\title{
Applications of Machine Learning and High-Performance Computing in the Era of COVID-19
}

\author{
Abdul Majeed ${ }^{1, *}$ (I) and Sungchang Lee ${ }^{2, *}$ \\ 1 Department of Computer Engineering, Gachon University, Seongnam 13120, Korea \\ 2 School of Information and Electronics Engineering, Korea Aerospace University, Goyang 10540, Korea \\ * Correspondence: ab09@gachon.ac.kr or abdulmajid09398@kau.kr (A.M.); sclee@kau.ac.kr (S.L.); \\ Tel.: +82-10-9503-9597 (A.M.)
}

Citation: Majeed, A.; Lee, S. Applications of Machine Learning and High- Performance Computing in the Era of COVID-19. Appl. Syst. Innov. 2021, 4, 40. https://doi.org/ $10.3390 /$ asi4030040

Academic Editor: Andrey Chernov

Received: 14 May 2021

Accepted: 28 June 2021

Published: 30 June 2021

Publisher's Note: MDPI stays neutral with regard to jurisdictional claims in published maps and institutional affiliations.

Copyright: (c) 2021 by the authors. Licensee MDPI, Basel, Switzerland. This article is an open access article distributed under the terms and conditions of the Creative Commons Attribution (CC BY) license (https:// creativecommons.org/licenses/by/ $4.0 /)$.

\begin{abstract}
During the ongoing pandemic of the novel coronavirus disease 2019 (COVID-19), latest technologies such as artificial intelligence (AI), blockchain, learning paradigms (machine, deep, smart, few short, extreme learning, etc.), high-performance computing (HPC), Internet of Medical Things (IoMT), and Industry 4.0 have played a vital role. These technologies helped to contain the disease's spread by predicting contaminated people/places, as well as forecasting future trends. In this article, we provide insights into the applications of machine learning (ML) and high-performance computing (HPC) in the era of COVID-19. We discuss the person-specific data that are being collected to lower the COVID-19 spread and highlight the remarkable opportunities it provides for knowledge extraction leveraging low-cost ML and HPC techniques. We demonstrate the role of ML and HPC in the context of the COVID-19 era with the successful implementation or proposition in three contexts: (i) ML and HPC use in the data life cycle, (ii) ML and HPC use in analytics on COVID-19 data, and (iii) the general-purpose applications of both techniques in COVID-19's arena. In addition, we discuss the privacy and security issues and architecture of the prototype system to demonstrate the proposed research. Finally, we discuss the challenges of the available data and highlight the issues that hinder the applicability of ML and HPC solutions on it.
\end{abstract}

Keywords: COVID-19; machine learning; high-performance computing; person-specific data; healthcare; Internet of Medical Things; infectious diseases

\section{Introduction}

The novel coronavirus disease 2019 (COVID-19) has drastically changed the era of information gathering, processing, analytics, use, distribution, and removal. Due to the COVID-19 pandemic, a huge amount of data is collected by various organizations to estimate the probability of exposure or tracing infected individuals [1]. In addition, this pandemic has increased the reliance on digital technologies to analyze people's mobility and monitor people's compliance with the quarantine guidelines [2]. In addition, many companies have helped governments with innovative technologies to control the COVID19 crisis [3]. The role of advanced technologies has been dominant in effectively handling this pandemic. Many countries have better control of the pandemic by utilizing technology and human resources simultaneously [4]. The innovative features of each technology have helped to curb the disease spread. The unique features of this pandemic such as estimating the possibility of infection, the identification of potentially contaminated places, contact tracing, mobility analysis, flow modeling, infected people's information sharing, data analytics, high-risk-zone geofencing, compliance monitoring, trends prediction, estimating the likelihood of this pandemic's end, heterogeneous source data fusion, and the adoption of remote technologies have increased the utilization of digital technology. We present the taxonomy of the epidemic features that demand the utilization of advanced techniques such as machine learning (ML) and high-performance computing (HPC) in Figure 1. These 
features are unique to the COVID-19 era and require the utilization of advanced technologies to curb the spread. Through the utilization of ML and HPC, significant mitigation and control of this pandemic can be achieved.
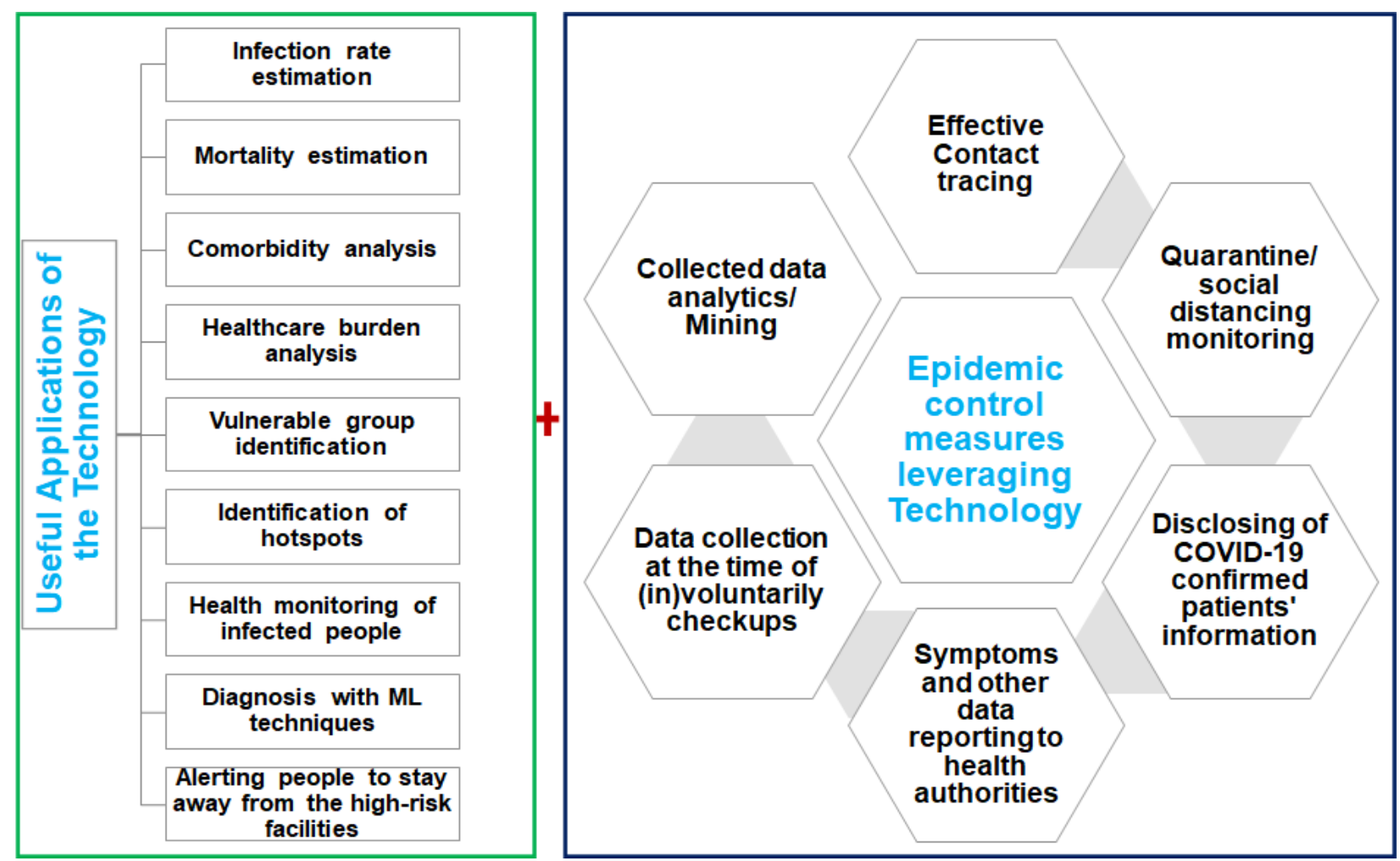

Figure 1. Taxonomy of the epidemic features that demand the utilization of advanced techniques such as ML and HPC.

Despite the enormous benefits of technologies in the COVID-19 era, the adoption of digital solutions has been low, mainly due to privacy issues [5]. Furthermore, some countries have adopted digital solutions by underestimating privacy requirements, and significant results have been obtained in terms of disease control. Moreover, people's anxiety and worries remain higher in such countries. For instance, in South Korea, credit card data, CCTV data, mobile signals, and random calls were adopted to trace the contacts of infected people. Similarly, China used advanced decision support systems to control the disease. In some countries, mobile apps were employed to find potently suspected patients. Through a detailed synthesis of the literature and analysis of multiple available apps and reports, we provide the statistics of ten countries in terms of higher adoption of AI over a period of almost one and half years in Figure 2. Furthermore, we provide insights into the investment in technology by companies around the world to combat the crisis caused by the ongoing pandemic [6].

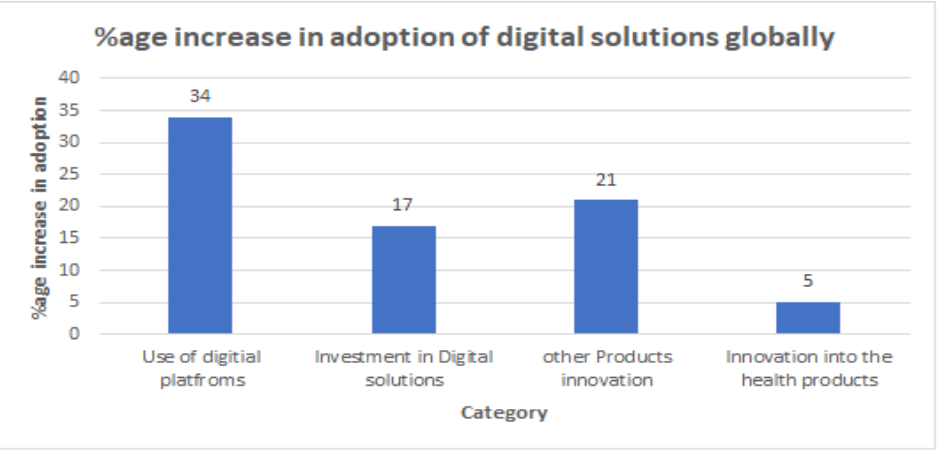

(a)

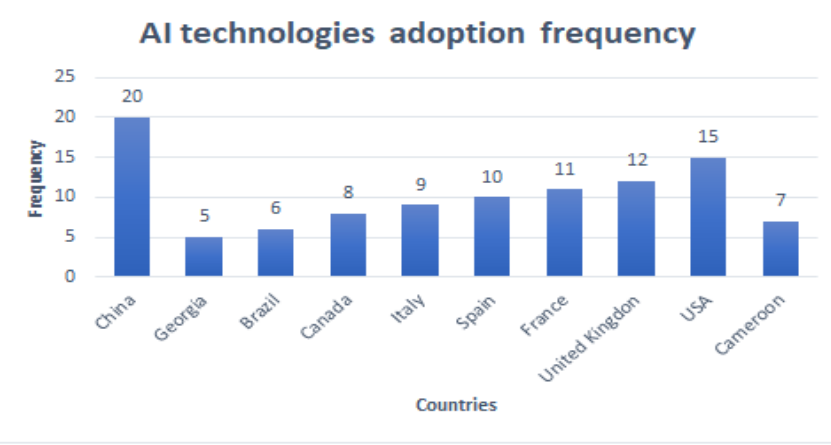

(b)

Figure 2. Statistics about (a) investment in digital solutions and (b) the adoption of AI around the world. 
To date, many studies have provided coverage of AI applications in the era of COVID19. However, these studies have mainly focused on the general applications of the technology in the education sector, industry areas, tourism sector, etc. However, a concrete overview of ML and HPC usage in the COVID-19 context, and their usefulness in the mitigation and control of this pandemic, has not been discussed in previous studies. To address these issues, this study covers the applications of ML and HPC considering epidemic features (i.e., epidemic control measures, data life cycle in epidemic systems, and general application of ML and HPC). More specifically, we highlight the role, need, and utility of ML and HPC technologies in the COVID-19 era. To this end, a compact overview of the fidelity of ML and HPC techniques in the era of COVID-19 is demonstrated with practical examples. Through this concise perspective, we hope to provide a solid foundation for future research in the COVID-19 area.

The rest of this perspective is organized as follows. Section 2 provides an insight into the data of individuals collected and processed in the COVID-19 era from heterogeneous sources. Section 3 highlights the effectiveness of the ML and HPC techniques in the COVID19 era. Section 4 discusses the challenges of technology adoption due to data issues, security and privacy issues, a prototype system to demonstrate the research, state-of-the-art studies and their key findings, and potential research directions. Finally, the study is concluded in Section 5 .

\section{Insights on Data Collection and Analytics Opportunities in the COVID-19 Era}

In recent years, data have been regarded as an oil of the economy, as they can assist nations and countries in countless ways, including human behavior analysis, recommendations, policy planning, and improving the living standards of people, to name a few. The latest technologies are very good at finding insights from large-scale data [7]. We highlight the data collected and processed in the COVID-19 era and corresponding analytics opportunities offered by them in Figure 3.

\section{Overview of Data sources, Digital solutions and Analytics opportunities in the COVID-19 Era}

\begin{tabular}{|c|c|c|}
\hline Internet of things (loT) Data & Smart Phone Applications & $\begin{array}{l}\text { [Contact tracing, symptoms reporting, } \\
\text { telemedicine, mobility pattern analysis] }\end{array}$ \\
\hline $\begin{array}{l}\text { Search Engine, E-Commerce, } \\
\text { and Navigation Data }\end{array}$ & $\begin{array}{l}\text { Machine/Deep Learning and } \\
\text { Natural Language Processing }\end{array}$ & $\begin{array}{l}\text { [Web-based epidemic intelligence tools, } \\
\text { sound analysis, diagnosis of COVID-19] }\end{array}$ \\
\hline Mobile Devices Data & $\begin{array}{c}\text { Social Media, Cell Phones, and } \\
\text { Online Searches }\end{array}$ & $\begin{array}{l}\text { [SMS Messages and Instant Messages, } \\
\text { alerts, targeted public health messages] }\end{array}$ \\
\hline Social Media Data & $\begin{array}{l}\text { Wearable Devices and Low- } \\
\text { Cost Sensors }\end{array}$ & $\begin{array}{l}\text { [Quarantine management, symptoms } \\
\text { reporting, mobility analysis, telemedicine] }\end{array}$ \\
\hline $\begin{array}{c}\text { Genetic and other historical } \\
\text { Data }\end{array}$ & $\begin{array}{l}\text { Visualization Tools/Platforms } \\
\text { for Health Information }\end{array}$ & $\begin{array}{l}\text { [Interactive dashboard, interactive } \\
\text { geospatial maps, risky zones plot, hotspots] }\end{array}$ \\
\hline $\begin{array}{c}\text { Medical Images and Sounds } \\
\text { Data }\end{array}$ & $\begin{array}{l}\text { Security and Privacy } \\
\text { Preserving Technologies }\end{array}$ & $\begin{array}{l}\text { [Zero knowledge proof, confidential } \\
\text { analytics, functional encryption, anonymity] }\end{array}$ \\
\hline Demographics Data & $\begin{array}{l}\text { Digital Diagnostics and Smart } \\
\text { Detection }\end{array}$ & $\begin{array}{l}\text { [Video observation for social distancing, } \\
\text { fingerprint for temperature estimation] }\end{array}$ \\
\hline (a) Different data sources & (b) Digital solutions & (c) Remarkable opportunities for analytics. \\
\hline
\end{tabular}

Figure 3. Conceptual overview of the data sources, digital solutions, and analytics (DDA) in the COVID-19 era. 
Apart from these techniques, Big Data analytics on existing data, such as commodities data, have profound benefits in separating the vulnerable population [8]. In addition, the collection of facilities' visit log data can be employed in individual profiling for mobility analysis. Despite the comprehensive analytics given in Figure 3, we provide the following analytics functions that can be exploited to curb the disease spread.

- $\quad$ Finding the most exposed people through location analysis (e.g., church/moviegoers).

- Extraction of people where physical presence for a job is mandatory.

- Identification of people working in high-risk zones.

- $\quad$ Alerting people when they have a high chance of getting COVID-19.

- Cluster identification by fusing multiple source data.

- Analyzing contact among people and predicting the risk score based on multicriteria.

- Identifying close contacts in a privacy-preserving manner.

- Analyzing the dynamics of the disease with respect to age groups.

- Identifying lethal combinations of the diseases to lower mortality chances.

- Providing the probability of infection score based on multiple parameters.

- Finding the connection of the diseases and demographics to better guide people to improve their health.

- Analyzing the dynamics of the disease with ambient conditions.

- Analyzing the correlation between symptoms and ICU transfer for resource planning.

- Predicting discharge dates based on demographics and comorbidity data.

The analytics documented above open up the era of research with new data that have not been analyzed before. With the help of the latest technologies, all of the above tasks can be achieved with sufficient accuracy to improve healthcare. By utilizing the above functions, the burden of healthcare can be significantly reduced. Furthermore, automated diagnosis and prescriptions can be provided with ease.

\section{Effectiveness of ML and HPC Techniques in the COVID-19 Era}

The combined utilization of ML and HPC can pave the way for improved healthcare and the effective mitigation and control of COVID-19. In the recent past, these techniques have demonstrated effectiveness in controlling the disease. HPC is very helpful in processing largescale data of individuals. We present the role of ML and HPC in the COVID-19 era in three contexts: in the data management life cycle, in analytics, and in general-purpose applications.

\subsection{ML and HPC Techniques' Role in the Data Management Life Cycle}

The data life cycle (DLC) is an important building block of knowledge-based applications. It has seven phases, and each phase has unique functions to be performed on the data. We provide an overview of the phases of the DLC in Table 1.

Table 1. Concise overview of the data life cycle employed to combat the COVID-19.

\begin{tabular}{ccc}
\hline Phase Number & Phase Name & Tasks Performed in a Respective Phase \\
\hline 1. & Collection & Data are collected from relevant people. \\
2. & Storage & Data are stored in databases for later use \\
3. & Preprocessing & Data are cleaned for later use. \\
4. & Analytics & Data are processed with ML and HPC for insights. \\
5. & Usage & Data are used for desired purposes. \\
6. & Distribution & Data are shared for research purposes. \\
7. & Archival & Data are archived for needs-based use. \\
\hline
\end{tabular}

The list of phases provided in Table 1 is generic, and they can be adopted for COVID-19 applications/systems. We highlight the use of ML and HPC in the above-mentioned phases of the DLC in Figure 4. ML techniques are mostly used for extracting desired knowledge from data. In contrast, HPC techniques can be useful for storing intermediate results or processing large-scale data. 


\section{Utilization of the ML and HPC in data life cycle of the COVID-19 Era}

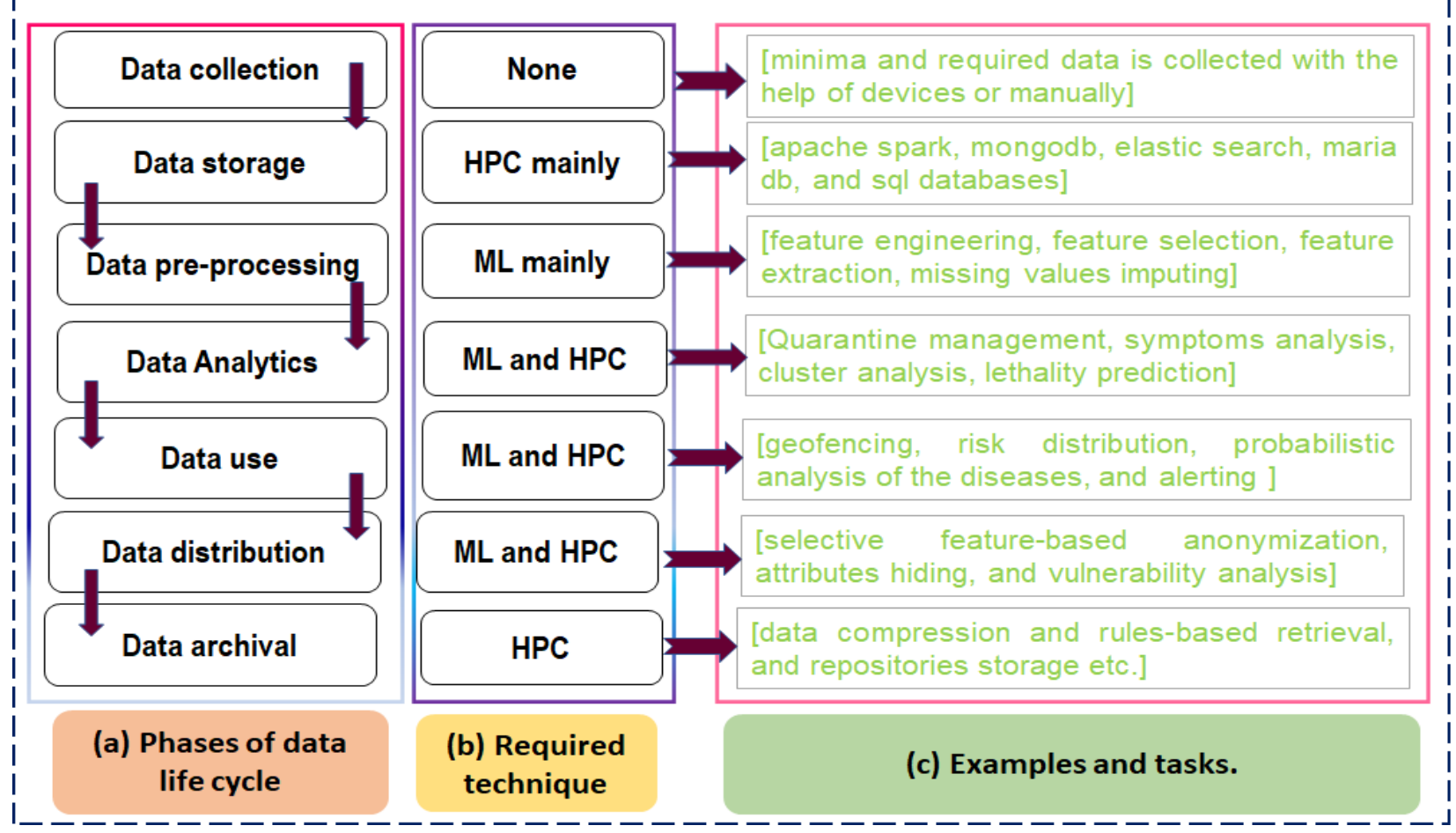

Figure 4. Role of ML and HPC in the data life cycle to fight COVID-19 through technology.

\subsection{Role of ML and HPC Techniques in Data Analytics in the COVID-19 Era}

Data have become a crucial part of knowledge-based applications. They unlock the knowledge enclosed in underlying data. The latest analytics techniques with hyperparameter tuning functionalities have proven successful in many applications. We present the role of ML and HPC in analytics in the COVID-19 era in Figure 5. ML techniques can assist in knowledge extraction. For instance, they can be widely used in identifying patients with and without COVID-19. ML techniques can also be used to rank the comorbidities that can lead to deaths, ICU admission, oxygen need, etc. They can also be used to separate people that can easily become targets of COVID-19 due to their work nature or hygiene practices. In addition, they can assist in trends analysis, privacy-preserving analytics, and data distribution across organizations. In contrast, HPC techniques have more utility from an administration point of view; for example, community clustering involves large-scale data processing. In this regard, HPC techniques are handy to load, process, hold intermediate results, and deliver results to interested parties. Therefore, both these techniques play a critical role in the COVID-19 era. In some cases, both techniques are jointly used to perform relevant tasks. The potential discussion for more useful features of both technologies can be determined from the latest studies [9-12]. 


\section{Utilization of the ML and HPC for Data Analytics on COVID-19 related data}

\begin{tabular}{|c|c|}
\hline $\begin{array}{l}\square \text { Identification of COVID-19 using chest CT } \\
\text { images, coughing patterns etc. } \\
\square \text { Detecting of COVID-19 in suspected patients } \\
\text { with sign and symptoms } \\
\square \text { COVID-19 quantitative chest CT assessment } \\
\text { for spot identifications } \\
\square \text { Screening, tracking and predicting the } \\
\text { current and future COVID-19 patients } \\
\square \text { Cluster identification based on population } \\
\text { density } \\
\square \text { Vulnerable groups identifications } \\
\square \text { Breathing pattern analysis for oxygen } \\
\text { situation analysis } \\
\square \text { Heterogeneous sources data fusion for } \\
\text { insight analysis } \\
\square \text { Social media analytics for forming the } \\
\text { taxonomy of symptoms } \\
\square \text { Rumours detection for the infectious } \\
\text { diseases. }\end{array}$ & $\begin{aligned} \rightarrow & \text { Heterogeneous and large-scale data support } \\
& \text { for processing and insight finding } \\
\rightarrow & \text { Handling diversities in the collected data for } \\
& \text { informative analysis } \\
\rightarrow & \text { Provision of the effective support for large } \\
& \text { scale data distribution that is invaluable for } \\
& \text { conducting effective research } \\
\rightarrow & \text { Providing support in processing data from } \\
& \text { multiple contexts. } \\
\rightarrow & \text { Insight analysis through interactive } \\
& \text { visualizations holding million or trillion } \\
& \text { records } \\
\rightarrow & \text { Providing support for an online machine } \\
& \text { learning execution in third parties } \\
& \text { infrastructure } \\
\rightarrow & \text { Handling variations in the data to support } \\
& \text { embedded knowledge extraction. } \\
\rightarrow & \text { Higher scalability to arrange the computing } \\
& \text { resources for effective processing of the } \\
& \text { large-scale data of patients. }\end{aligned}$ \\
\hline & (b) High Performance Computing (HPC) \\
\hline
\end{tabular}

Figure 5. Role of ML and HPC in data analytics to fight with COVID-19 through technology.

\subsection{Role of ML and HPC Techniques in the General-Purpose Applications Related to the} COVID-19 Era

Despite the specific applications in diagnostic and analytics, ML and the HPC techniques can be used for general-purpose applications. We summarize the potential generalpurpose applications of ML and HPC as follows.

- Creating awareness about COVID-19 through sentiment analysis and recommending health tips.

- Regional- and community-level analysis of electronic health records of confirmed/ suspected COVID-19 cases.

- Providing medical statistics about COVID-19 symptoms from real case data.

- Providing the locality of hospitals and clinics.

- Increasing data utility by recommending/sharing results on cell phones.

- Processing and utilization of cell phone signal data for people identification.

- Extracting and fusing multistyle data for accurate analysis.

- Combining multimodel results to erase people's anxiety on results' credibility.

- Analyzing and sharing human behavior for vulnerable links identification in the community.

- Analyzing the technology implications on society through relevant features.

There exist multiple general applications of both HPC and ML that are assisting societies in multiple ways $[13,14]$. Furthermore, both techniques can be used for measuring and analyzing the data of multiple institutions for better analysis of the COVID-19 pandemic.

\section{Discussion}

This section briefly discusses the data-related challenges that hinder the effective utilization of ML and HPC techniques, privacy and security issues in the COVID-19 context, a prototype system to demonstrate the effectiveness of the AI and HPC techniques, and promising future research directions.

\subsection{Data-Related Challenges That Hinder the Effective Utilization of ML and HPC Techniques}

Although ML and HPC techniques are excellent at finding hidden knowledge from data, multiple issues related to collected data, such as unavailability of benchmark datasets, error-prone data, raw data, and limited access to related data due to privacy issues, are the main barriers in the adoption of these techniques. In addition, privacy and security issues are 
further important challenges that limit the data reusability and results dissemination across organizations [15]. We identified twelve technical features that can impact the adoption of the latest technologies (i.e., AI, ML, HPC etc). The challenges are listed below.

- Unavailability of the benchmark datasets.

- Data availability at a very limited scale.

- Data contain a substantial number of missing/incomplete records.

- Data are highly unreliable, as they are collected through privacy enforcement.

- Data are in inconsistent styles; therefore. fusion is not easy.

- Data are highly skewed and sparse.

- Data are related to only some communities (i.e., minorities).

- Data cannot provide sufficient insights about the disease dynamics.

- Data cannot assist in making effective policies to separate vulnerable communities (e.g., comorbidity data are not shared mostly).

- Data do not provide all dynamics related to COVID-19.

- Data do not support full-scale adoption of ML and HPC techniques due to privacy and security issues.

In addition to the key challenges cited above, selection of the appropriate ML and HPC techniques is also very challenging. Hence, before applying any ML methods, understanding the data structure and related problems that can arise in analytics is of paramount importance.

\subsection{Privacy and Security Issues}

Due to the urgency of the situation to manage and develop solutions for the COVID19 pandemic, information sharing in large volumes between international and national organizations (e.g., people with the virus (also known as electronic health records) and treatment data, contact tracing data, multimedia data produced by digital applications such as Zoom, Meet, Webex, Teams, etc.) has become inevitable. Furthermore, information sharing is an important building block for the realization of Big Data, and at the same time, it can lead to infringements on individual privacy. Due to the COVID-19 pandemic, different kinds of privacy and security issues have been reported in media around the world due to massive data collection and processing to curb the spread of COVID-19. Hence, the need to preserve people's privacy has become more urgent than ever. Due to privacy issues at a large scale, we may require more robust organizational, constitutional, and technical measures to address the security and privacy issues of digital applications in the COVID-19 era. Furthermore, heavy reliance on digital solutions, such as IoE and IoT sensors, Internet, SN, 5G networks, and HPC-based computing powers, will make the problem even worse. The ongoing pandemic has highlighted that existing privacy and security mechanisms are not sufficient to address the privacy and security implications emerging from the ongoing pandemic. They require a brand-new start to adhere to more stern data protection laws and regulations (e.g., HIPAA, IPA, GDPR, etc.) around the world to comfort people regarding their privacy requirements. We highlight the privacy and security issues of emerging Big Data applications and other digital technologies that are extensively used during the era of the COVID-19 pandemic in Figure 6. Recently, numerous pertinent solutions focusing on privacy preservation have been proposed [16-21]. However, general public trust in digital solutions use remained very low, mainly due to privacy issues. Therefore, the privacy and security issues presented in Figure 6 require a brand-new start to develop more practical solutions to serve the community in effective ways. 
Comprehensive overview of Privacy \& Security Issues in Pre and COVID-19 Era

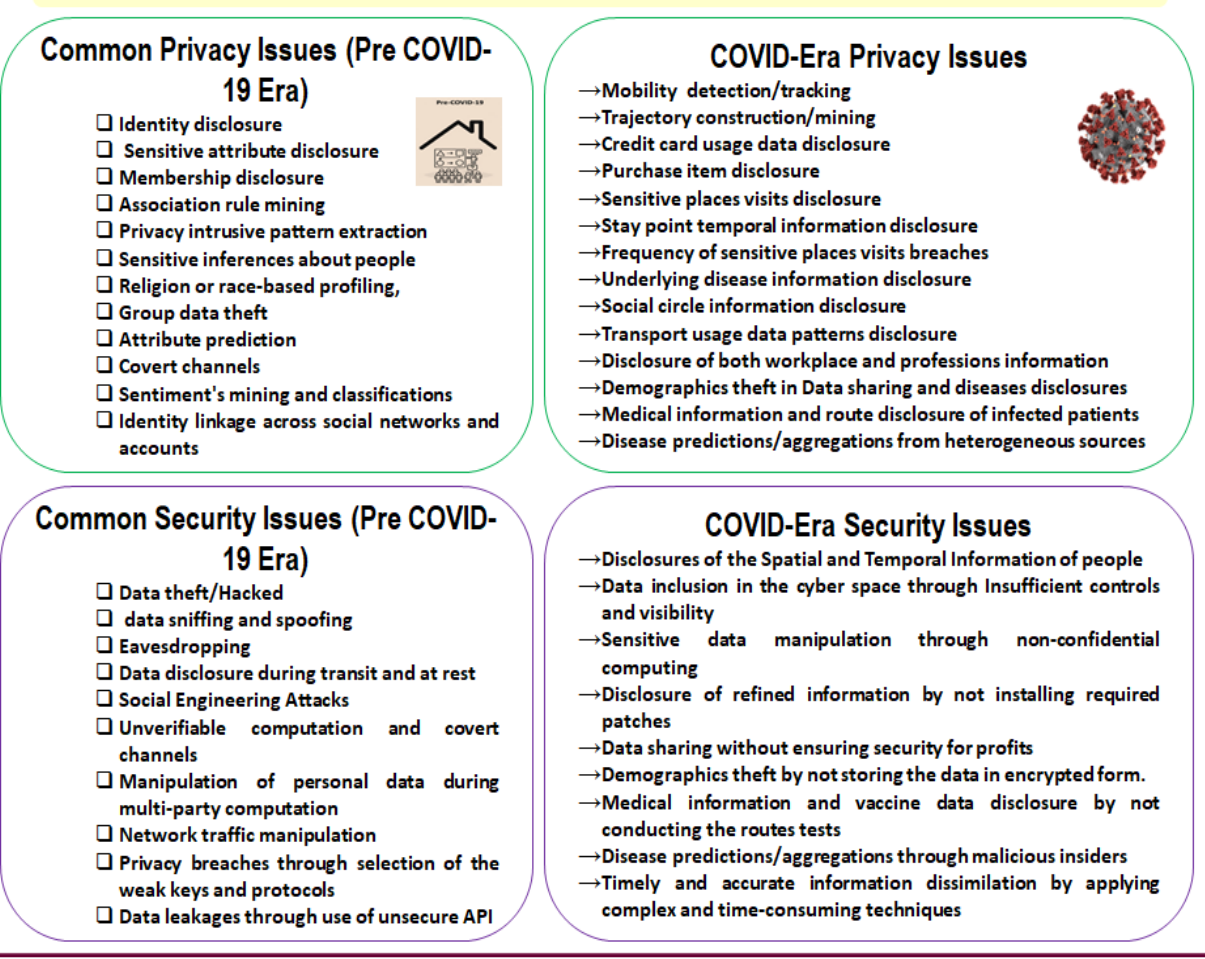

Figure 6. Comprehensive overview of the privacy and security issues prior to and in the COVID-19 era.

\subsection{Architecture of Prototype System to Demonstrate the Effectiveness of AI and HPC Techniques in COVID Context}

From the start of this unprecedented pandemic, a number of digital solutions have been proposed for different utilities such as digital contact tracing [22], IoT-based generic models for other infectious disease and COVID-19 suspects tracing [23], predictions of COVID-19 malignant progression [24], medical AI systems [25], expert systems for clinical guidelines [26], and real-time data based COVID-19 screening using wearable sensors data [27], to name a few. After a detailed synthesis of these systems, we found that all existing solutions were proposed to address one aspect of the COVID-19 pandemic. To address this deficiency, we designed an architecture of the prototype system to demonstrate the effectiveness of the AI and HPC techniques in the COVID-19 context and the need for such a system. The need for an AI- and HPC-powered system is demonstrated in Figure 7a, and the proposed prototype system architecture is shown in Figure $7 \mathrm{~b}$. The proposed prototype has three main modules that can be enhanced more in the future. The main modules, along with their brief description, are presented below.

- Data collection: In this module, data are collected from relevant individuals and authentic sources such as healthcare departments and agencies. We identify three main sources of data that can contribute effectively to this pandemic, such as real-environment data (including social distancing, mask information, nature of contact with other people, spatiotemporal-based activities data, social circle information, etc.), authentic healthcare departments data (including previous disease history, travel information, ambient data, etc.), and IoT and SN data (including people behaviors).

- Data processing and analytics leveraging ML and HPC techniques: In this module, data are processed with multiple ML techniques for the possibility of infections estimation, spread predictions, and spatiotemporal information correlation for COVID19 spread hidden route identifications. The role of HPC is to provide support during data processing and storing intermediate results at a massive scale.

- Results utilization for disease mitigation and control: In this module, results are disseminated to the relevant parties (e.g., general people, health officials, and government 
officials). The sharing of results with relevant parties accurately and timely contributes significantly to disease mitigation and control.

\section{Top challenges}

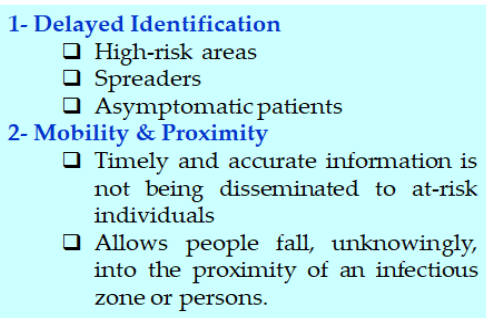

Timely and accurate information is not being disseminated to at-risk individuals

๑ Allows people fall, unknowingly, into the proximity of an infectious zone or persons.

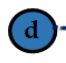

Bringing it All Together

- Proactively identify and geo fence

- Supervise the spreaders

Know the math of COVID-19

- Planning effective interventions

\section{AI \& HPC-driven solution}

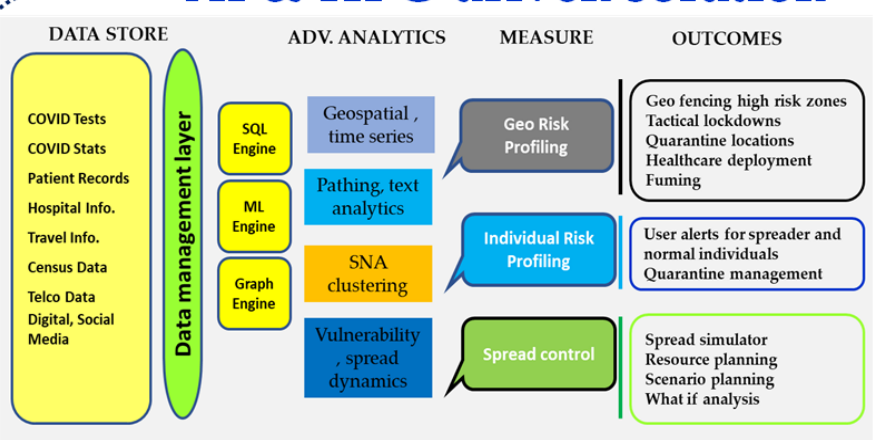

\section{C) Business outcomes}

1- Early identification of the COVID-19 case

2- Informed mobility of patients and spreaders

3- Effective healthcare outreach and administration

(a) Need of the AI \& HPC powered system to fight with the COVID-19

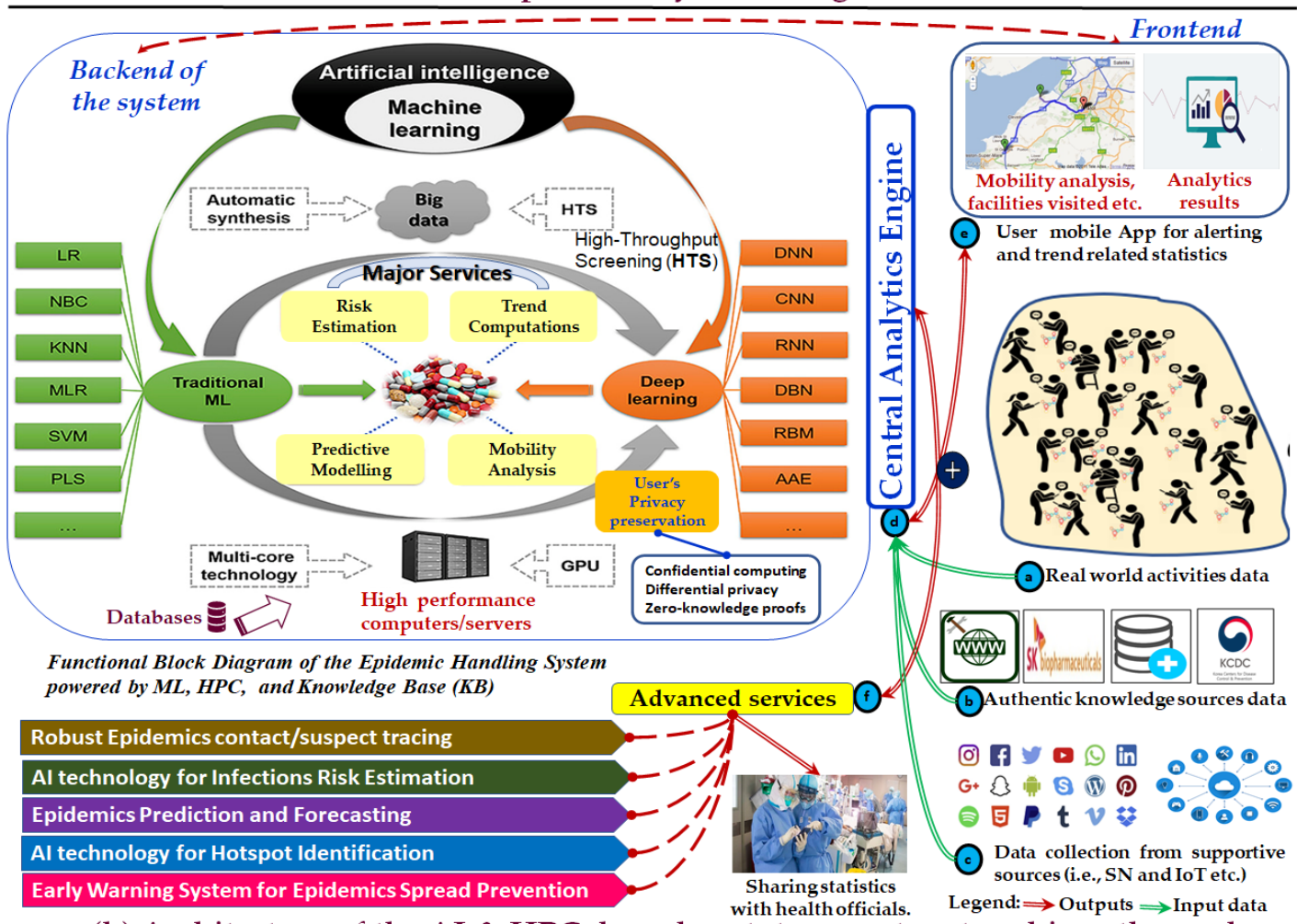

(b) Architecture of the AI \& HPC-based prototype system to achieve the goal

Figure 7. (a) Need for an ML- and HPC-based system in the era of COVID-19 and (b) architecture of the ML- and HPC-based prototype system to fight with the COVID-19.

4.4. Comprehensive Overview of the Current State-of-the-Art Research Involving the Use of ML and HPC Methods in the Era of COVID-19

In this subsection, we provide a comprehensive overview regarding the current state-ofthe-art (SOTA) studies involving the use of ML and HPC methods in the era of COVID-19. To date, a substantial number of SOTA studies have provided coverage regarding the use of ML and HPC methods in the era of COVID-19. We discuss the latest SOTA studies, their findings, and how these findings can address the clinical unmet needs in the COVID-19 in Table 2. 


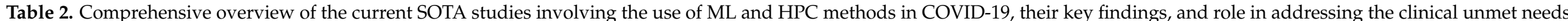
in the era of COVID-19.

\begin{tabular}{|c|c|c|c|}
\hline \multirow{2}{*}{ Category } & SOTA Studies & Discussions about Findings and Role in Addressing the Clinical Unmet Needs in COVID-19. & \\
\hline & Study Details & Key Findings of Each SOTA Study & Role in Addressing the Clinical Unmet Needs in the COVID-19 Era \\
\hline \multirow{19}{*}{ ML } & Pinter et al. [28] & Predictions of mortality rate and time series of infected individuals & Outbreak's modeling and mortality trends analysis \\
\hline & Magar et al. [29] & Virus-antibody sequence analysis and Identification of potential patients & Robust identification of antibodies that potentially inhibit COVID-19 \\
\hline & Aminu et al. [30] & Accurate detection of people with COVID-19 with limited data & Effective for the reliable diagnosis of COVID-19 \\
\hline & Zeng et al. [31] & Forecasting of patient survival probability & Age-group-based mortality analysis to provide care to elderly people. \\
\hline & Shah et al. [32] & COVID-19 detection from $X$-ray images & Help in diagnosing potential suspects as early as possible to mitigate the deadly disease \\
\hline & Ashraf et al. [33] & Predict the severity of disease or chances of death & Significant contribution in separating vulnerable groups for ample care \\
\hline & Prakash et al. [34] & Impact analysis of various policies employed to control the disease & Guidance for effective strategies that can help control the spread \\
\hline & Ullah et al. [35] & Classification of patients with and without COVID-19 & Lowering the healthcare burden of patient diagnosis \\
\hline & Rathod et al. [36] & Effective crisis preparedness and management along with authorities' responses and mitigation strategies. & Assistance in healthcare workers' burden analysis \\
\hline & Rathod et al. [37] & Detection of abnormal data for effective analysis & Resource planning and accurate diagnosis \\
\hline & Hu et al. [39] & Feasible analysis model for the treatment and diagnosis of COVID-19 & Effective identifications of key symptoms and medicines for different syndromes \\
\hline & Singh et al. [40] & Reduce the high false-negative results of the RT-PCR & Effectively handles the sensitivity issue that is associated with RT-PCR \\
\hline & Peddinti et al. [41] & Detection of COVID-19 cases in public places & Helps officials in the accurate and faster diagnosis of the virus \\
\hline & Saverino et al. [42] & Changes implementation in rehabilitation services & Staff satisfaction and stress reduction during pandemic times \\
\hline & Lella et al. [43] & Respiratory sound classification for potential patient identification & Classification of asthma sounds, COVID-19 sounds, and regular healthy sounds \\
\hline & Malla et al. [44] & Real-time sentiment analysis of COVID-19 tweets & Tweets prediction related to similar types of infectious diseases in the future \\
\hline & Ibrahim et al. [45] & Accurately diagnosing COVID-19 patients and analyzing severity level & Detecting COVID-19 patients and classifying the severity degree from chest CT slices \\
\hline & Roland et al. [46] & Blood-test-based identification of patients with COVID-19 and estimate the mortality risk & Automatic scanning of COVID-19 in a cost-effective way without any additional efforts \\
\hline & Gros et al. [47] & Accurate estimates of the cumulative medical load of COVID-19 outbreaks & Understanding the outbreak dynamics and predicting future cases and fatalities \\
\hline \multirow{5}{*}{ HPC } & Hack et al. [48] & Promising treatments, including the virus' protein structure and attack mechanisms analysis, and resource planning & Accelerate the science needed to develop treatments and strategies to combat COVID-19 \\
\hline & West et al. [49] & COVID-19 spread analysis among different populations and effective therapeutic response & Virus transmission analysis at a very large scale \\
\hline & LeGrand et al. [50] & Drug discovery for targeting the proteins of the COVID-19 virus responsible for the current pandemic & Drug discovery for COVID-19 by analyzing large-scale docking campaigns \\
\hline & Mulholland et al. [53] & Provides insights into the inner workings and mechanisms of the molecules of COVID-19 & Can assist in suggesting potential drug candidates \\
\hline & Zaki et al. [54] & Identify drug/lead candidate for better inhibitory activity against the main protease of COVID-19. & Useful to develop a therapeutic agent for COVID-19. \\
\hline \multirow{3}{*}{ Hybrid } & Pathak et al. [55] & Innovative solutions for restricting COVID-19 spread & Guidelines for pharmaceutical companies to devise better cures \\
\hline & Bhati et al. [56] & Combines HPC and ML to accelerate drug discovery & Target proteins analysis to identify lead compounds \\
\hline & Bharadwaj et al. [57] & Development of potential vaccines at a much diminished time and lower cost & Tackling pandemics and overcoming the crisis with computing intelligence \\
\hline
\end{tabular}




\subsection{Promising Research Directions for Future in the Era of COVID-19}

In the future, it will be interesting to devise good models to make sense of the data, accurate predictions, and forecasting leveraging heterogeneous sources data, variations handling in epidemics data, and accurate trends analysis through model fusions. In addition, proposing COVID-19-specific models and new evaluation criteria(s) to evaluate the effectiveness of these models is another promising research direction. Furthermore, designing and developing robust and accurate privacy-preserving models for individual privacy preservation is imperative. To this end, the development of a prototype system that can address the privacy implications of all epidemic control measures demonstrated in Figure 1 is handy for the well-being of the community.

\section{Conclusions}

This paper presented the role of the latest technologies (i.e., ML and HPC) in the fight against the unanticipated challenge of COVID-19. Specifically, we presented an overview of the epidemic features that require ML and HPC techniques to serve humankind in an effective way by mitigating the pandemic through technology. We described the multitude of heterogeneous types of data collected in the COVID-19 era and the remarkable opportunities they offer when analyzed with advanced ML and HPC techniques. We highlighted the effectiveness of ML and HPC techniques in the mitigation and control of the COVID-19 pandemic through their unique use in a variety of applications. We provided potential research directions and challenges in the technical adoption of ML and HPC due to data issues (i.e., unavailability, sparsity, imprecise, data poisoning, etc.). We believe that this unique study provides a solid foundation for future studies in this area in relation to pandemic features and corresponding data. The main contributions of this study are given as follows.

- It presents an overview of epidemic features that require ML and HPC techniques to serve humankind in an effective way by controlling the pandemic through technology.

- It describes the multitude of heterogeneous types of data collected in the COVID-19 era and the remarkable opportunities they offer when analyzed with advanced ML and HPC techniques.

- It highlights the effectiveness of ML and HPC techniques in the mitigation and control of the COVID-19 pandemic through their unique use in three contexts.

- It provides potential research directions and challenges that hinder the adoption of ML and HPC due to data issues (i.e., availability, sparsity, data poisoning, etc.)

- It discusses the privacy and security issues and architecture of a prototype system to demonstrate the proposed research.

- To the best of our knowledge, this is the first work to provide a concise overview of the ML and HPC techniques used in the COVID-19 era with respective data in loop.

In addition to the key contributions given above, a vibrant area of research is developing flexible anonymization methods that can easily be tuned, based on the original data characteristics or circumstances to foster data reusability. In addition, using ML and HPC techniques to process large-scale data in a privacy-preserving manner is of paramount importance for future endeavors.

Author Contributions: Conceptualization, A.M. and S.L.; Writing, A.M.; Data curation, A.M.; Methodology, A.M.; Supervision, S.L.; Project administration, S.L.; Funding acquisition, S.L. All authors have read and agreed to the published version of the manuscript.

Funding: This research was supported by National Research Foundation of Korea (NRF) Grant funded by the Korean Government (Ministry of Science and ICT) NRF-2020K1A3A1A47110830.

Institutional Review Board Statement: Not applicable.

Informed Consent Statement: Not applicable. 
Data Availability Statement: The statistical data used to support the findings of this study are included within the article.

Conflicts of Interest: The authors declare no conflict of interest.

\section{References}

1. Hale, T.; Angrist, N.; Goldszmidt, R.; Kira, B.; Petherick, A.; Phillips, T.; Webster, S.; Cameron-Blake, E.; Hallas, L.; Majumdar, S.; et al. A global panel database of pandemic policies (Oxford COVID-19 Government Response Tracker). Nat. Hum. Behav. 2021, 5, 529-538. [CrossRef]

2. Mbunge, E.; Akinnuwesi, B.; Fashoto, S.G.; Metfula, A.S.; Mashwama, P. A critical review of emerging technologies for tackling COVID-19 pandemic. Hum. Behav. Emerg. Technol. 2021, 3, 25-39. [CrossRef]

3. Bijan, M. Developed Technologies and Active Startup Companies in Dealing with COVID-19 Pandemic in Iran. J. Control 2021, 14, 97-105.

4. Mbunge, E.; Dzinamarira, T.; Fashoto, S.G.; Batani, J. Emerging technologies and COVID-19 Digital Vaccination Certificates and Passports. Public Health Pract. 2021, 2, 100136. [CrossRef] [PubMed]

5. Wen, H.; Zhao, Q.; Lin, Z.; Xuan, D.; Shroff, N. A study of the privacy of covid-19 contact tracing apps. In International Conference on Security and Privacy in Communication Systems; Springer: Cham, Switzerland, 2020; pp. 297-317.

6. Apedo-Amah, M.C.; Avdiu, B.; Cirera, X.; Cruz, M.; Davies, E.; Grover, A.; Iacovone, L.; Kilinc, U.; Medvedev, D.; Maduko, F.O.; et al. Unmasking the impact of covid-19 on businesses: Firm level evidence from across the world. Policy Res. Work. Pap. 2020. [CrossRef]

7. Wieringa, J.; Kannan, P.K.; Ma, X.; Reutterer, T.; Risselada, H.; Skiera, B. Data analytics in a privacy-concerned world. J. Bus. Res. 2021, 122, 915-925. [CrossRef]

8. Mirsky, J.B.; Horn, D.M. Chronic disease management in the COVID-19 era. Am. J. Manag. Care 2020, $26,329-330$.

9. Cortés, U.; Cortés, A.; Garcia-Gasulla, D.; Pérez-Arnal, R.; Álvarez-Napagao, S.; Àlvarez, E. The ethical use of high-performance computing and artificial intelligence: Fighting COVID-19 at Barcelona Supercomputing Center. AI Ethics 2021, 1-16. [CrossRef]

10. Al-Emran, M.; Al-Kabi, M.N.; Marques, G. A Survey of Using Machine Learning Algorithms During the COVID-19 Pandemic. In Emerging Technologies During the Era of COVID-19 Pandemic; Springer: Cham, Switzerland, 2021; Volume 348, pp. 1-8.

11. Swapnarekha, H.; Behera, H.S.; Nayak, J.; Naik, B. Role of intelligent computing in COVID-19 prognosis: A state-of-the-art review. ChaosSolitons Fractals 2020, 138, 109947. [CrossRef]

12. Elnaggar, A.; Heinzinger, M.; Dallago, C.; Rihawi, G.; Wang, Y.; Jones, L.; Gibbs, T.; Feher, T.; Angerer, C.; Steinegger, M.; et al. ProtTrans: Towards Cracking the Language of Life's Code Through Self-Supervised Deep Learning and High Performance Computing. arXiv 2020, arXiv:2007.06225.

13. Arora, A.; Chakraborty, P.; Bhatia, M.P.S. Problematic use of digital technologies and its impact on mental health during COVID-19 pandemic: Assessment using machine learning. Emerg. Technol. Dur. Era COVID-19 Pandemic 2021, 348, $197-221$.

14. Zivkovic, M.; Bacanin, N.; Venkatachalam, K.; Nayyar, A.; Djordjevic, A.; Strumberger, I.; Al-Turjman, F. COVID-19 cases prediction by using hybrid machine learning and beetle antennae search approach. Sustain. Cities Soc. 2021, 66, 102669. [CrossRef]

15. Majeed, A. Attribute-centric anonymization scheme for improving user privacy and utility of publishing e-health data. J. King Saud Univ. Comput. Inf. Sci. 2019, 31, 426-435. [CrossRef]

16. Michael, V. Privacy is not the problem with the Apple-Google contact-tracing toolkit. Guard, 2020. Available online: https: //www.theguardian.com/commentisfree/2020/jul/01/apple-google-contact-tracing-app-tech-giant-digital-rights (accessed on 5 May 2021).

17. Abdul, M. Towards Privacy Paradigm Shift Due to the Pandemic: A Brief Perspective. Inventions 2021, 6, 24.

18. Sowmiya, B.; Abhijith, V.S.; Sudersan, S.; Sundar, R.S.J.; Thangavel, M.; Varalakshmi, P. A Survey on Security and Privacy Issues in Contact Tracing Application of Covid-19. SN Comput. Sci. 2021, 2, 1-11. [CrossRef] [PubMed]

19. Mallory, K. Public Health, Big Tech, and Privacy Squaring the Contact-Tracing Circle. COVID-19 and the Information Space Boosting the Democratic Response, Global Insights. p. 44. Available online: https://www.ned.org/wp-content/uploads/2021/0 1/Global-Insights-COVID-19-Information-Space-Boosting-Democratic-Response-1.pdf-page=44 (accessed on 5 May 2021).

20. McGraw, D.; Mandl, K.D. Privacy protections to encourage use of health-relevant digital data in a learning health system. NPJ Digit. Med. 2021, 4, 1-11. [CrossRef] [PubMed]

21. Shin, S.-Y. Privacy Protection and Data Utilization. Healthc. Inform. Res. 2021, 27, 1-2. [CrossRef] [PubMed]

22. Kleinman, R.A.; Merkel, C. Digital contact tracing for COVID-19. CMAJ 2020, 192, E653-E656. [CrossRef]

23. Garg, L.; Chukwu, E.; Nasser, N.; Chakraborty, C.; Garg, G. Anonymity preserving IoT-based COVID-19 and other infectious disease contact tracing model. IEEE Access 2020, 8, 159402-159414. [CrossRef]

24. Fang, C.; Bai, S.; Chen, Q.; Zhou, Y.; Xia, L.; Qin, L.; Gong, S.; Xie, X.; Zhou, C.; Tu, D.; et al. Deep learning for predicting COVID-19 malignant progression. Med. Image Anal. 2021, 72, 102096. [CrossRef]

25. Wang, B.; Jin, S.; Yan, Q.; Xu, H.; Luo, C.; Wei, L.; Zhao, W.; Hou, X.; Ma, W.; Xu, Z.; et al. AI-assisted CT imaging analysis for COVID-19 screening: Building and deploying a medical AI system. Appl. Soft Comput. 2021, 98, 106897. [CrossRef] [PubMed]

26. Banjar, H.R.; Alkhatabi, H.; Alganmi, N.; Almouhana, G.I. Prototype Development of an Expert System of Computerized Clinical Guidelines for COVID-19 Diagnosis and Management in Saudi Arabia. Int. J. Environ. Res. Public Health 2020, 17, 8066. [CrossRef] [PubMed] 
27. Mukhtar, H.; Rubaiee, S.; Krichen, M.; Alroobaea, R. An IoT Framework for Screening of COVID-19 Using Real-Time Data from Wearable Sensors. Int. J. Environ. Res. Public Health 2021, 18, 4022. [CrossRef] [PubMed]

28. Pinter, G.; Felde, I.; Mosavi, A.; Ghamisi, P.; Gloaguen, R. COVID-19 Pandemic Prediction for Hungary; a Hybrid Machine Learning Approach. Mathematics 2020, 8, 890. [CrossRef]

29. Magar, R.; Yadav, P.; Farimani, A.B. Potential neutralizing antibodies discovered for novel corona virus using machine learning. Sci. Rep. 2021, 11, 5261. [CrossRef]

30. Aminu, M.; Ahmad, N.A.; Noor, M.H.M. Covid-19 detection via deep neural network and occlusion sensitivity maps. Alex. Eng. J. 2021, 60, 4829-4855. [CrossRef]

31. Zeng, W.; Gautam, A.; Huson, D.H. On the Application of Advanced Machine Learning Methods to Analyze Enhanced, Multimodal Data from Persons Infected with COVID-19. Computation 2021, 9, 4. [CrossRef]

32. Shah, P.M.; Ullah, F.; Shah, D.; Gani, A.; Maple, C.; Wang, Y.; Shahid, A.; Abrar, M.; Islam, S.U. Deep GRU-CNN model for COVID-19 detection from chest $X$-rays data. IEEE Access 2021. [CrossRef]

33. Ashraf, I.; Alnumay, W.S.; Ali, R.; Hur, S.; Bashir, A.K.; Zikria, Y.B. Prediction Models for COVID-19 Integrating Age Groups, Gender, and Underlying Conditions. Comput. Mater. Contin. 2021, 67, 3009-3044. [CrossRef]

34. Prakash, P.N.S.; Hariharan, B.; Kaliraj, S.; Siva, R.; Vivek, D. The impact of various policy factors implemented for controlling the spread of COVID-19. Mater. Today Proc. 2021. [CrossRef]

35. Ullah, S.I.; Salam, A.; Ullah, W.; Imad, M. COVID-19 lung image classification based on logistic regression and support vector machine. In European, Asian, Middle Eastern, North African Conference on Management E Information Systems; Springer: Cham, Switzerland, 2021; pp. 13-23.

36. Rathod, P.; Usoro, A. Benchmarking Machine Learning Approaches to Predict the Uncertainties of Pandemic Disease: An Explorative Study. In Proceedings of the 7th Annual International Conference on Information Technology and Economic Development, Gregory University, Uturu, Nigeria, 16-18 November 2020.

37. Al Meshal, R.A.K.H. The Impact of COVID-19 on Arabian Gulf Countries Using the Classical Machine Learning Methods. MECSJ. 2021. Available online: https://www.mecsj.com/uplode/images/photo/The_impact_of_COVID-19_on_Arabian_Gulf_ countries_using_the_Classical_Machine_Learning_Methods.pdf (accessed on 30 June 2021).

38. Rashed, E.A.; Hirata, A. One-Year Lesson: Machine Learning Prediction of COVID-19 Positive Cases with Meteorological Data and Mobility Estimate in Japan. Int. J. Environ. Res. Public Health 2021, 18, 5736. [CrossRef]

39. Hu, F.; Huang, M.; Sun, J.; Zhang, X.; Liu, J. An analysis model of diagnosis and treatment for COVID-19 pandemic based on medical information fusion. Inf. Fusion 2021, 73, 11-21. [CrossRef] [PubMed]

40. Singh, D.; Kumar, V.; Kaur, M. Densely connected convolutional networks-based COVID-19 screening model. Appl. Intell. 2021, 51, 3044-3051. [CrossRef]

41. Peddinti, B.; Shaikh, A.; Bhavya, K.R.; Nithin Kumar, K.C. Framework for Real-Time Detection and Identification of possible patients of COVID-19 at public places. Biomed. Signal Process. Control 2021, 68, 102605. [CrossRef]

42. Saverino, A.; Baiardi, P.; Galata, G.; Pedemonte, G.; Vassallo, C.; Pistarini, C. The Challenge of Reorganizing Rehabilitation Services at the Time of COVID-19 Pandemic: A New Digital and Artificial Intelligence Platform to Support Team Work in Planning and Delivering Safe and High Quality Care. Front. Neurol. 2021, 12, 643251. [CrossRef] [PubMed]

43. Lella, K.K.; Pja, A. Automatic COVID-19 disease diagnosis using 1D convolutional neural network and augmentation with human respiratory sound based on parameters: Cough, breath, and voice. AIMS Public Health 2021, 8, 240. [CrossRef] [PubMed]

44. Malla, S.; Alphonse, P.J.A. COVID-19 outbreak: An ensemble pre-trained deep learning model for detecting informative tweets. Appl. Soft Comput. 2021, 107, 107495. [CrossRef]

45. Ibrahim, M.R.; Youssef, S.M.; Fathalla, K.M. Abnormality detection and intelligent severity assessment of human chest computed tomography scans using deep learning: A case study on SARS-COV-2 assessment. J. Ambient Intell. Humaniz. Comput. 2021, 1-24. [CrossRef]

46. Roland, T.; Boeck, C.; Tschoellitsch, T.; Maletzky, A.; Hochreiter, S.; Meier, J.; Klambauer, G. Machine Learning based COVID-19 Diagnosis from Blood Tests with Robustness to Domain Shifts. medRxiv 2021. [CrossRef]

47. Gros, C.; Valenti, R.; Schneider, L.; Gutsche, B.; Markovi'c, D. Predicting the cumulative medical load of COVID-19 outbreaks after the peak in daily fatalities. PLoS ONE 2021, 16, e0247272. [CrossRef]

48. Hack, J.J.; Papka, M.E. The US High-Performance Computing Consortium in the Fight Against COVID-19. Comput. Sci. Eng. 2020, 22, 75-80. [CrossRef]

49. West, J. Large-Scale Scientific Computing in the Fight Against COVID-19. Comput. Sci. Eng. 2021, 23, 89-92. [CrossRef]

50. LeGrand, S.; Scheinberg, A.; Tillack, A.F.; Thavappiragasam, M.; Vermaas, J.V.; Agarwal, R.; Larkin, J.; Poole, D.; SantosMartins, D.; Solis-Vasquez, L.; et al. GPU-accelerated drug discovery with docking on the summit supercomputer: Porting, optimization, and application to COVID-19 research. In Proceedings of the 11th ACM International Conference on Bioinformatics, Computational Biology and Health Informatics, Virtual Event, USA, 21-24 September 2020; pp. 1-10.

51. Vermaas, J.V.; Sedova, A.; Baker, M.; Boehm, S.; Rogers, D.; Larkin, J.; Glaser, J.; Smith, M.; Hernandez, O.; Smith, J. Supercomputing Pipelines Search for Therapeutics Against COVID-19. Comput. Sci. Eng. 2020, 23, 7-16. [CrossRef]

52. Pérez-Moraga, R.; Forés-Martos, J.; Suay-García, B.; Duval, J.; Falcó, A.; Climent, J. A COVID-19 Drug Repurposing Strategy through Quantitative Homological Similarities Using a Topological Data Analysis-Based Framework. Pharmaceutics 2021, 13, 488. [CrossRef] 
53. Mulholland, A.J.; Amaro, R.E. COVID19-Computational Chemists Meet the Moment. J. Chem. Inf. Model. 2020, 60, 5724-5726. [CrossRef]

54. Zaki Magdi, E.A.; Al-Hussain, S.A.; Masand, V.H.; Akasapu, S.; Bajaj, S.O.; El-Sayed, N.N.E.; Ghosh, A.; Lewaa, I. Identification of Anti-SARS-CoV-2 Compounds from Food Using QSAR-Based Virtual Screening, Molecular Docking, and Molecular Dynamics Simulation Analysis. Pharmaceuticals 2021, 14, 357. [CrossRef] [PubMed]

55. Pathak, N.; Deb, P.K.; Mukherjee, A.; Misra, S. IoT-to-the-Rescue: A Survey of IoT Solutions for COVID-19-like Pandemics. IEEE Internet Things J. 2021. [CrossRef]

56. Bhati, A.P.; Wan, S.; Alfè, D.; Clyde, A.R.; Bode, M.; Tan, L.; Titov, M.; Merzky, A.; Turilli, M.; Jha, S.; et al. Pandemic Drugs at Pandemic Speed: Accelerating COVID-19 Drug Discovery with Hybrid Machine Learning-and Physics-based Simulations on High Performance Computers. arXiv 2021, arXiv:2103.02843.

57. Bharadwaj, K.K.; Srivastava, A.; Panda, M.K.; Singh, Y.D.; Maharana, R.; Mandal, K.; Singh, B.S.M.; Singh, D.; Das, M.; Murmu, D.; et al. Computational intelligence in vaccine design against COVID-19. In Computational Intelligence Methods in COVID-19: Surveillance, Prevention, Prediction and Diagnosis; Springer: Singapore, 2021; pp. 311-329. 